POS PROCEEDINGS

\title{
Observation of the Identical Rigidity Dependence of the Primary Cosmic Rays Helium, Carbon and Oxygen fluxes by the Alpha Magnetic Spectrometer on the International Space Station
}

\author{
Zhili Weng ${ }^{* \dagger}$ \\ Massachusetts Institute of Technology, Cambridge, USA \\ E-mail: zhili.weng@cern.ch
}

\begin{abstract}
We report on the observation of new and unexpected properties of primary cosmic rays, $\mathrm{He}, \mathrm{C}$, and $\mathrm{O}$, measured in the rigidity (momentum/charge) range $2 \mathrm{GV}$ to $3 \mathrm{TV}$ with 90 million helium, 8.4 million carbon, and 7.0 million oxygen nuclei collected by the Alpha Magnetic Spectrometer on the International Space Station during the first 5 years of operation. Unexpectedly, above 60 $\mathrm{GV}$, these three spectra have identical rigidity dependence. They all deviate from a single power law above $200 \mathrm{GV}$ and harden in an identical way. These are new and unexpected properties of primary cosmic rays and they are challenging current model of CR production, acceleration, and propagation.
\end{abstract}

The 39th International Conference on High Energy Physics (ICHEP2018)

4-11 July, 2018

Seoul, Korea

${ }^{*}$ Speaker.

${ }^{\dagger}$ On Behalf of the AMS Collaboration 
Helium, carbon, and oxygen are primary cosmic ray, mainly produced and accelerated in astrophysical sources. They carry information on the source and the propagation history. Precise knowledge of their spectra in the GV - TV rigidity region provides important insights to the origin, acceleration, and propagation of cosmic rays in the Galaxy [1].

This proceeding reports on the the observation of the identical rigidity dependence of the helium, carbon and oxygen fluxes by the Alpha Magnetic Spectrometer (AMS) on the International Space Station[2].

\section{Precision Measurements with AMS-02 Detector}

The layout and description of the AMS detector are presented in Ref. [2, 3, and references therein]. The key elements used in this measurement are the permanent magnet, 9 layers of silicon tracker, and the four planes of time of flight (TOF) scintillation counters. Together, the tracker and the magnet measure the rigidity $R$ of charged cosmic rays. The maximum detectable rigidity (MDR) of 3.2 TV for helium, 3.7 TV for carbon, and 3.4 TV for oxygen over the $3 \mathrm{~m}$ lever arm from $L 1$ to $L 9$. Each layer of the tracker provides an independent measurement of the charge $Z$. Overall, the inner tracker has a resolution of $\Delta Z / Z=3.5 \%$ for helium, $2 \%$ for carbon, and $1.5 \%$ for oxygen. Two of the TOF planes are located above the magnet (upper TOF) and two planes are below the magnet (lower TOF). The overall velocity $(\beta=v / c)$ resolution has been measured to be $\Delta \beta / \beta^{2}=0.02$ for helium and $\Delta \beta / \beta^{2}=0.01$ for carbon and oxygen nuclei. The two upper planes and two lower planes are combined to provide two independent measurement of the charge, each with an accuracy $\Delta Z / Z$ better than $4 \%$.

The detailed data analysis is presented in Ref[2]. In total $90 \times 10^{6}$ helium events, $8.4 \times 10^{6}$ carbon and $7.0 \times 10^{6}$ oxygen nuclei are selected for this analysis. With large amount of statistics, extensive study on systematic uncertainties is a crucial part of the analysis. Systematic errors include the uncertainties in the background estimations, the trigger efficiency, the geomagnetic cutoff factor, the acceptance calculation, the rigidity resolution function, and the absolute rigidity scale. The total error is $\approx 3 \%$ at $100 \mathrm{GV}$ for both the carbon and oxygen fluxes and $\approx 1.5 \%$ at 100 GV for the helium flux.

\section{Observation of Identical Rigidity Dependence of He, C, O Fluxes}

Figure 1 shows the (a) helium, (b) carbon, and (c) oxygen fluxes as functions of rigidity, together with earlier measurements of the helium [4] and carbon [5] fluxes. As seen in Fig. 1(a), the AMS measurement of the helium flux is distinctly different from the results of Ref. [4] which shows sharp spectrum shape change. As seen in Fig. 1(b), the AMS measurement of the carbon flux is also distinctly different from the results of Ref. [5] which are $20-25 \%$ lower above $20 \mathrm{GV}$.

To examine the rigidity dependence of the fluxes, the variation of the flux spectral indices with rigidity was obtained in a model independent way. This is presented in Fig. 1(d). As seen, The magnitude and the rigidity dependence of the helium, carbon, and oxygen spectral indices are very similar and they all deviate from a single power law. In particular, all spectral indices are identical within the measurement errors above $60 \mathrm{GV}$ and all spectral indices harden with rigidity above $\sim 200 \mathrm{GV}$. 

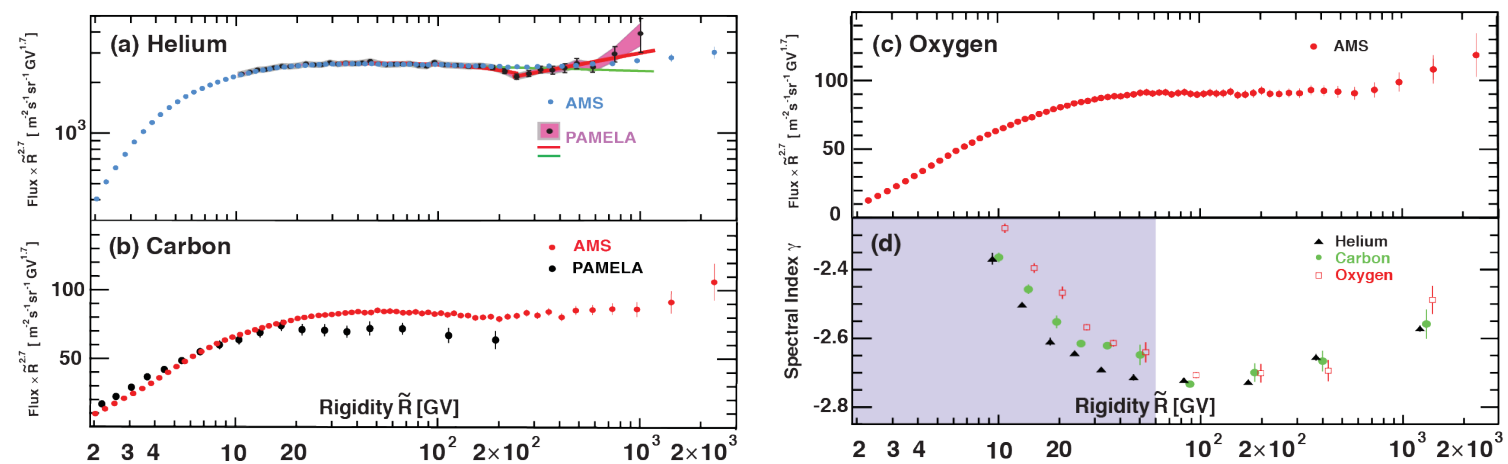

Figure 1: The AMS (a) helium, (b) carbon, and (c) oxygen fluxes multiplied by $\tilde{R}^{2.7}$ as functions of rigidity. (d) The dependence of the helium, carbon, and oxygen spectral indices on rigidity. In (d), for clarity, the horizontal positions of the helium and oxygen data points are displaced with respect to carbon.

To study in detail the difference between the rigidity dependence of the helium, carbon and oxygen fluxes, we present the flux ratio of helium flux to oxygen flux $(\mathrm{He} / \mathrm{O})$ and carbon flux to oxygen flux (C/O ratio) in Fig. 2.
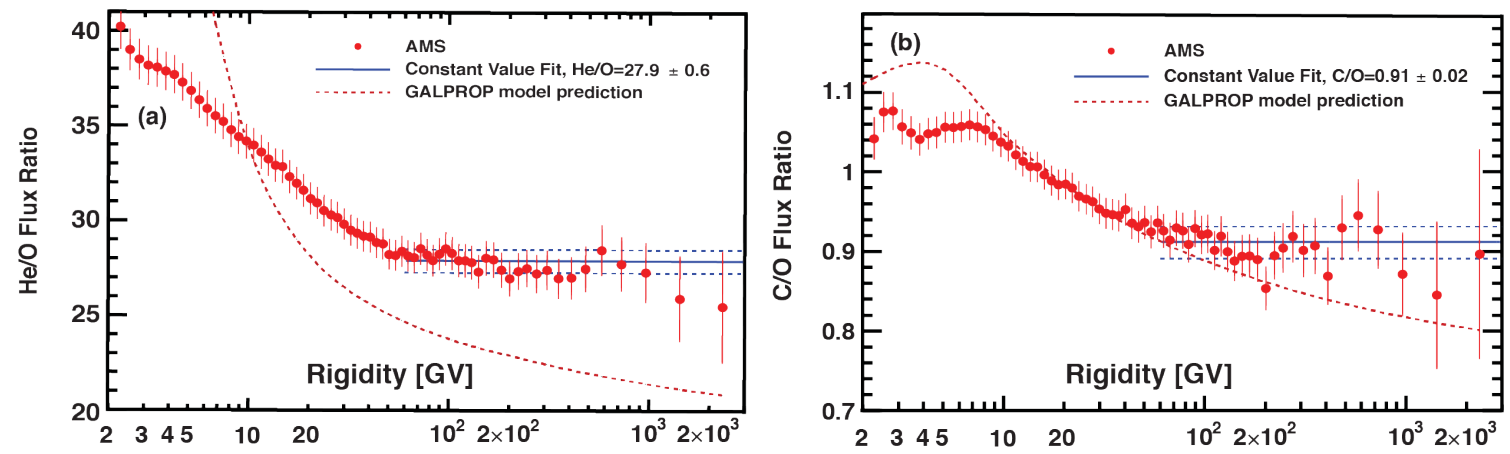

Figure 2: The (a) $\mathrm{He} / \mathrm{O}$ and (b) $\mathrm{C} / \mathrm{O}$ ratios as functions of rigidity compared with the GALPROP model predictions [6], dashed red curves.

As seen, above $60 \mathrm{GV}$, the ratio of the helium flux to the oxygen flux (He/O ratio) is well fit by a constant value of $27.9 \pm 0.6$, and the ratio of the carbon flux to the oxygen flux ( $\mathrm{C} / \mathrm{O}$ ratio) is well fit by a constant value of $0.91 \pm 0.02$. This is distinctly different from the GALPROP prediction of a decreasing $\mathrm{He} / \mathrm{O}$ and $\mathrm{C} / \mathrm{O}$ ratio at this rigidity range [6]. None of these unexpected observation can be explained by the current understanding of cosmic rays.

\section{Conclusion and Outlooks}

In conclusion, Precision measurements of the helium, carbon, and oxygen fluxes from $2 \mathrm{GV}$ to $3 \mathrm{TV}$ show that their fluxes all deviate from a single power law. As illustrated in Fig. 3, above $60 \mathrm{GV}$, the three fluxes have identical rigidity dependence. Their spectral indices all progressively harden above $200 \mathrm{GV}$. Detailed studies on the different rigidity behavior shows that above $60 \mathrm{GV}$, the helium to oxygen flux ratio is constant at $27.9 \pm 0.6$ and the carbon to oxygen flux ratio is constant at $0.91 \pm 0.02$. 


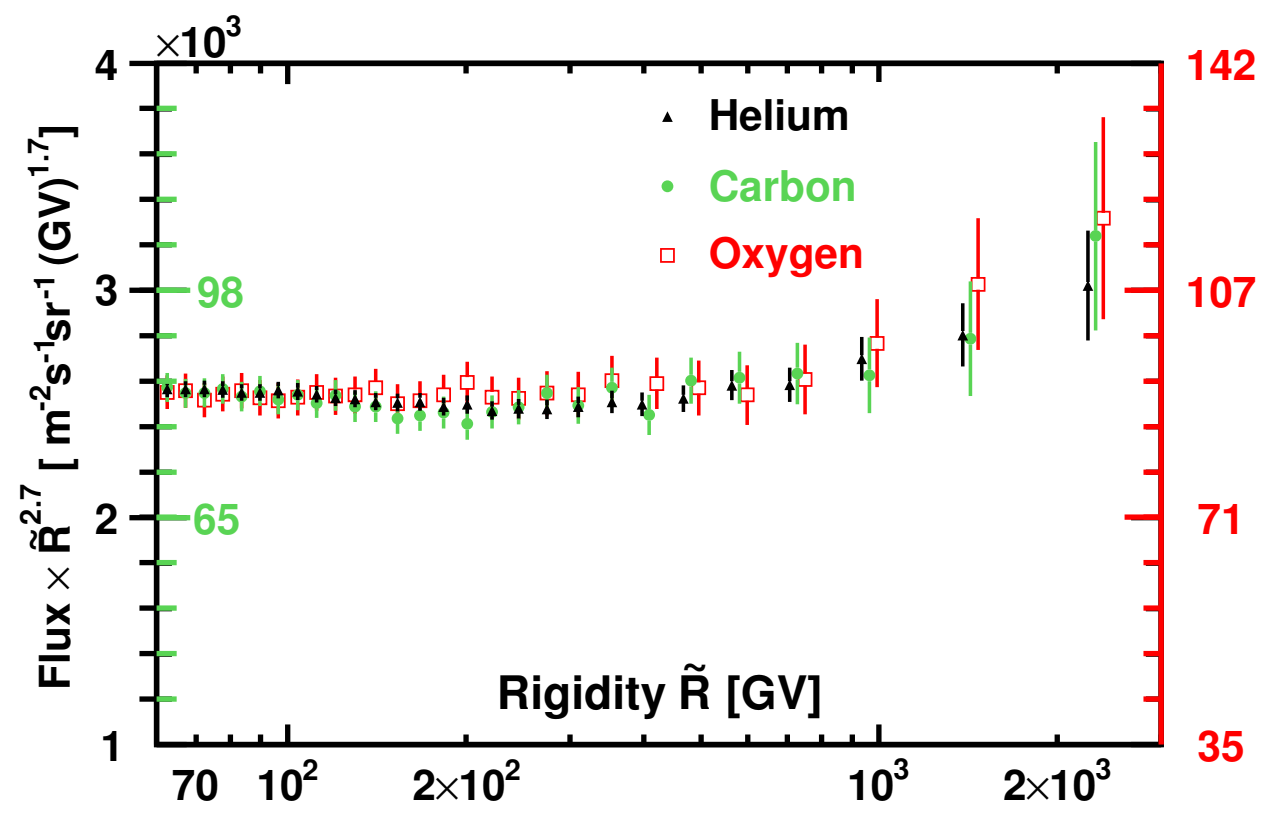

Figure 3: The rigidity dependence of the helium (left black axis), carbon (left green axis), and oxygen (right red axis) fluxes. Above $60 \mathrm{GV}$ the three fluxes have identical rigidity dependence.

These are new and unexpected properties of primary cosmic rays and they are challenging current model of CR production, acceleration, and propagation. Simultaneous measurement of many CR species is crucial for acquiring knowledge of cosmic ray physics and for the discovery of new phenomena. AMS will continue collecting data for the live time of the ISS, providing precision measurements of all cosmic rays nuclei up to Iron and beyond.

\section{References}

[1] I. A. Grenier, J. H. Black and A. W. Strong, Annu. Rev. Astron. Astrophys. 53, 199 (2015); P. Blasi, Astron. Astrophys. Rev. 21, 70 (2013); A. W. Strong, I. V. Moskalenko, and V. S. Ptuskin, Annu. Rev. Nucl. Part. Sci. 57, 285 (2007); A. Castellina and F. Donato, Astrop. Phys. 24, 146 (2005).

[2] M. Aguilar et al., Phys. Rev. Lett. 119, 251101 (2017).

[3] M. Aguilar et al., Phys. Rev. Lett. 110, 141102 (2013).

[4] O. Adriani, et al., Science 332, 69 (2011).

[5] O. Adriani et al., Astrophys. J. 791, 93 (2014).

[6] A. E. Vladimirov, et al., Comput. Phys. Comm. 182, 5 (2011), with parametrization from R. Trotta, et al., Astrophys. J. 729, 106 (2011). We thank Dr. I. V. Moskalenko for extensive discussions. 